\title{
CLASSES DE STEINITZ ET EXTENSIONS QUATERNIONIENNES
}

\author{
Richard Massy et Bouchaib Sodaïgui \\ Université de Valenciennes, Valenciennes, France.
}

\begin{abstract}
Let $k$ be a number field. We prove that the set of Steinitz classes of quadratic extensions $K / k$ which can be embedded in quaternionic extensions of degree 8, tamely or wildly ramified over $k$, is the full class group $\mathrm{Cl}(k)$ of $k$. Moreover, we provide explicit formulas to conutruct these quaternionic extensions.

Firyorus AND PHRASEs : Steinitz classes, explicit embedding problcm. tamcly or wildly ramified quaternionic extensions.

Running heAd titTle : Steinitz classes.
\end{abstract}




\section{Introduction}

Pour tout corps de nombres $F$, on note $O_{F}$ l'anneau des entiers de $F$. Soient $k$ un corps de nombres et $K / k$ une extension de degré $n$. L'anneau $O_{K}$ est un $O_{k}$-module sans torsion de rang $n$; il existe donc un idéal $I$ de $O_{k}$ tel que $O_{k} \simeq O_{k}^{n-1} \oplus I$ en tant que $O_{k}$-module. La classe de $I$ dans le group $\mathrm{Cl}(k)$ des classes de $k$ est appelée la classe de Steinitz de l'extension $K / k$ on de l'anneau $O_{K^{\prime}}$, et on la note $\mathrm{Cl}\left(\mathrm{O}_{K^{\circ}}\right)$.

$\mathrm{D}^{\circ}$ apròs [3 ou [15]. tout élénient de $\mathcal{C} l(k)$ est réalisable comme classe de Steinit $z$ d'une extension quadratique de $k$. Pour un nombre premier impair $l$, signalons que Long [8] a montré le résultat suivant: l'ensemble des classes de Steinitz des extensions cycliques de $k$ de degré $l^{r}(r \geq 1)$, et modérément ramifiées, est un sous-groupe de $\mathcal{C} l(k)$.

Dans ce papier. on appelle "extension quaternionienne de degré 8" une extension galoisienne de groupe de Galois isomorphe au groupe quaternionien $H_{8}$. On décrit un ensemble de classes de Steinitz d' extensions assujetties à une condition de plongement. Précisément, on décrit l'ensemble des classes de Steinitz des extensions quadratiques de $k$ qui se plongent dans des extensions cluaternioniennes de degré 8 modérément ou sauvagement ramifiées. Do phus. on fournit des formules explicites de construction de ces extensions quatemionicmeste.

\section{1.. Enoncé des résultats}

(On démontre section 2 les résultats suivants :

Théorème 11 Ponl tout corps de nombres $k$, l'ensemble des élénients de $\mathcal{C l}(k)$ réalisables comme classes de Steinitz d'extensions quadratiques de $k$, modérément (resp. sauvagement) ramifiées, et plongeables dans des extensions quaternioniennes de degré 8 sur $k$, modérément (resp. sauvagement) ranifiées, est égal au groupe $\mathcal{C l}(k)$.

Théorème 1.2 Soit $k$ un corps de nombres.

(1) Soit $k / k$ une extension quadratique modérément ramifiée. Le corps $k$ se plonge dans une extension quaternionienne de degré 8 sur $k$ si et seulement si les places réellos de $k$ se prolongent en des places réelles de $k$. 
(2) Suil $L=h(\sqrt{ } a \cdot \sqrt{ } b) / h$ unc extension biquadratique bicyclique, modériment ramificic. de groupe de Galois de générateurs $\sigma$ et $T$ définis para

$$
\sigma(\sqrt{a}) / \sqrt{ } a=\tau(\sqrt{b}) / \sqrt{b}=-1, \quad \sigma(\sqrt{b}) / \sqrt{b}=\tau(\sqrt{a}) / \sqrt{a}=1 .
$$

Soit $O_{2 . t}$ lanmean seni-local des ćlénents $a \in L$ de valuation $v_{\mathcal{D}}(a) \geq 0$ "n tous less idéanx premiers $\mathcal{D}$ de $L$ divisant $2 O_{L}$.

(2.1) Luxtersion $L / k$ se plonge dans whe extension quaternionienne, modérément ramifiée. $N / k$ de degré 8 si et seulement s'il existe. devix clincuts $y$ ot $z$ dans le groupe multiplicatif $1+2 \mathrm{O}_{2, L}$ tels que lon ait

$$
y \sigma(u)=1, \quad z \tau(z)=-1, \quad \sigma(z) / z=-\tau(y) / y .
$$

(2.2) Dans less conditions du (2.1), on peut prendre $N=L(\sqrt{x})$ avec pour a l'élónent suivant du groupe multiplicatif $1+4 O_{2, L}$

$$
x=\theta+y^{2} \sigma(\theta)+z^{2} \tau(\theta)+(y \sigma(z))^{2} \sigma \tau(\theta)
$$

$\sin \theta$ fist un rentice de $L$ de trace 1 sur $k: \theta \in O_{L}, \operatorname{Tr}_{L / k}(\theta)=1$.

Sitholies. 1) L'équivalence du (1) exprime que dans la Proposition III.2 de [2] (cf. infra), la seconde condition est toujours vérifiće lorsque l'extension $\mathrm{k} / \mathrm{k}$ est modérét'.

2) In (2) ronstruil unc solution $N / h$ modorment ramifice au probleme de

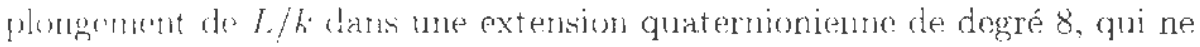

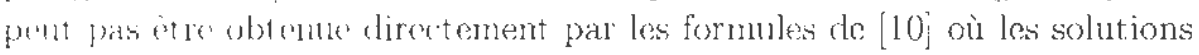
sont donnos sants eondition de ramifications.

\section{2.. Démonstrations}

Proposition 21 Solont $m \in k$ tel que $H=k(\sqrt{m})$ et $\Delta(K / k)$ le discriminaut a $\mathrm{K} / \mathrm{t}$.

(1) On peut écrire d'une nanière unique $m O_{k}=I(m)^{2} J$ où $I(m)$ est un idéal fractionaise de $O_{k}$, et $J$ un idéal entier de $O_{k}$ sans facteurs carrés.

(2) On a $\Delta(K / h)=J J^{\prime 2}$ où $J^{\prime}$ est un idćal cutier de $O_{k}$ divisant $2 O_{k}$. L. ixtension $\mathrm{K} / \mathrm{h}$ est modéróment ramifićn si of seulcment si $J^{\prime}=O_{k}$. 
(3) La chasice de Strinitz de $O_{k}$ est régale à la classe de lidéal $I(m)^{-1} J^{\prime}$ dans le grompe des classes de $k$ :

$$
\mathcal{C l}\left(O_{K}\right)=\mathcal{C l}\left(I(m)^{-1} J^{\prime}\right) .
$$

Démonstration. Les points (1) et (2) résultent de la théorie de Kummer et dia début de la section 2 de [3].

(3) Sulent $\mathrm{Y}$ lo réseau libre de $O_{k}$ dans $K$ de base $\{1, \sqrt{m}\}$, et $\Delta(X)$ son discriminant par rapport à la forme trace ; on a $\Delta(X)=4 \mathrm{~m}$. En utilisant la notion d'invariant relatif de deux réseaux (cf.[14] ; Chap.III $\$ 1, \S 2$ ), on montre sans difficulté qu'il existe un idéal fractionnaire $U$ de $O_{k}$ tel que $\Delta\left(K^{\prime} / h^{2}\right)=\Delta(X) U^{2}$ et $\mathcal{C l}\left(O_{K}\right)=\mathcal{C l}(U)$. La première égalité et le fait que $\Delta\left(K / k^{\prime}\right)=J J^{\prime 2}$ entrainent $m O_{k}=\left(2^{-1} U^{-1} J^{\prime}\right)^{2} J$. De l'unicité de $I(m)$ on déluit quo $I(m)=2^{-1} L^{-1} J^{\prime}$. et par comséquent

$$
\mathcal{C l}(L)=\mathcal{C l}\left(I(m)^{-1} J^{\prime}\right)=\mathcal{C l}\left(O_{K}\right) .
$$

Et ant domnée une extension quelconque $E / k$, soit le groupe multiplicatif

$$
\tau_{E}^{*}(n):=1+2^{n} O_{2, E} \quad(n \in \mathbb{N}-\{0\})
$$

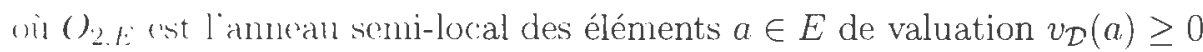
Pn trus lus idéaux premiers $\mathcal{D}$ de $E$ divisant $2 O_{E}$. On peut aussi définir $L_{E}^{+}(u)$ par la congruence mod $^{*}$ usuelle du corps de classes (cf. infra ou [1], p. 13) :

$$
U_{E}(n)=\left\{x \in E^{\times} / x \equiv 1 \bmod ^{*}\left(2^{n} O_{E}\right)\right\} .
$$

Pour tout corps intermédiaire $F, k \subseteq F \subseteq E$, on vérifie que

$$
\begin{aligned}
& U_{F}(n)=F^{\times} \cap U_{E}(n) \\
& U_{F}(2) \cap F^{\times 2}=U_{F}(1)^{2} .
\end{aligned}
$$

Le critère'suivant se déduit du $\$ 39$ de [6].

(2.4) Unc extension quadratique $E / F$ est modérément ramifiée si et sculcment s̆il existe un élément $x \in U_{F}(2)$ tel que $E=F(\sqrt{x})$.

\section{Démonstration du théorème 1.2}

(1) Soit $m \in k$ tel que $K=k(\sqrt{m})$. D'après [13] ou le Théorème IV.2 de $[2] . k / k$ est plongeable dans une extension quaternionienne sur $k$ de degré 8 si et seulement si $m$ est somme de trois carrés dans $k$, ce qui est équivalent par la Proposition III.2 de [2] aux deux conditions suivantes: (i) $m$ cst totalement positif, i.e pour tout plongement réel $\sigma$ de $k, \sigma(m)>0$; 
(ii) pour tout idéal prenier $\mathcal{B}$ de $O_{k}$ au dessus de 2 tel que $\left[k_{\mathcal{B}}: \mathbf{Q}_{2}\right]$ soit impair. - m n est pas un carré dans $k_{\mathcal{B}}$, où $k_{\mathcal{B}}$ est le complété de $k$ pour la place $\mathcal{B}$.

La condition du théorème 1.2 concernant le prolongement des places récllos de $h$ rst équivalente à (i): reste à montrer (ii).

(ii) Sorit $\mathcal{B}$ un idéal premier quelconque do $O_{k}$ au dessus de 2. Supposons yll" $-m$ sist un "arró lans $k_{\mathcal{B}}:-m=a^{2}\left(a \in h_{\mathcal{B}}\right)$, d'où $k_{\mathcal{B}}(\sqrt{m})=$ $k_{\mathcal{E}}(\sqrt{-1})$. de sorte que $k_{\mathcal{B}}(\sqrt{m})$ est le compositum des extensions $k_{\mathcal{B}}$ et Q.2 $(\sqrt{-1})$. On garde les mêmes notations pour les idéaux premiers et leurs complétés. Soit $\mathcal{P}$ (resp. $\mathcal{D}$ ) l'idéal premier au dessus de $\mathcal{B}$ (resp. 2) dans $k_{\mathcal{B}}(\sqrt{m})$ (resp. $\mathrm{Q}_{2}(\sqrt{-1})$ ). En notant par $e(/)$ les différents indices de ranification. on a par transitivité :

$$
(\mathcal{P} / 2)=e(\mathcal{P} / \mathcal{B}) \times e(\mathcal{B} / 2)=e(\mathcal{P} / \mathcal{D}) \times e(\mathcal{D} / 2)
$$

$L$ 'xxtension $K / k$ étant modérément ramifiée, on a $e(\mathcal{P} / \mathcal{B})=1$, et comme $e(\mathcal{D} / 2)=2$, c'est que $e(\mathcal{B} / 2)$ est pair. On en déduit que $\left[k_{\mathcal{B}}: \mathbf{Q}_{2}\right]$ est pair. Par conséquent, si l'on a $\left[k_{\mathcal{B}}: \mathbf{Q}_{2}\right]$ impair, $-m$ n'est pas un carré dans $k_{\mathcal{B}}$ : d'où (ii).

(2) Supposons que $L / k$ se plonge dans une extension quaternionienne muderément ramifiée $N / h$. Conme $N / L$ cst a fortiori modérée, il existe, dapros (2.4). un ćlément $x \in U_{L}(2)$ tel que $N=L(\sqrt{x})$. Du fait que $\gamma / h$ est galoisienne. on déduit alors par (2.3) que pour tout $\gamma \in \operatorname{Gal}(L / k)$, il existe $x_{\gamma} \in U_{L}(1)$ pour lequel $\gamma(x) / x=x_{\gamma}^{2}$. Soit maintenant $N^{\prime}=$ $L\left(\sqrt{x^{\prime}}\right) / k$ l'extension quaternionienne définie par les formules du Théorème 3 (B) de [10]: il existe $y^{\prime} \in L^{\times}, z^{\prime} \in L^{\times}$tels que

$$
y^{\prime} \sigma\left(y^{\prime}\right)=-1, \quad z^{\prime} \tau\left(z^{\prime}\right)=-1, \quad \sigma\left(z^{\prime}\right) / z^{\prime}=-\tau\left(y^{\prime}\right) / y^{\prime}
$$

ot lon a

$$
x^{\prime}=\sqrt{a b} \alpha z^{\prime}
$$

où $\alpha \in k(\sqrt{a})^{\times}$vérifie

$$
\alpha / \sigma(\alpha)=y^{\prime} \tau\left(y^{\prime}\right)
$$

De plus, en vertu du (3.3) de [10], il existe $c \in k^{\times}$et $l \in L^{\times}$pour lesquels

$$
x=c l^{2} x^{\prime}
$$

Il on résulto que

$$
x_{\sigma}= \pm \frac{\sigma(l)}{l} \frac{1}{y^{\prime}}, x_{\tau}= \pm \frac{\tau(l)}{l} \frac{1}{z^{\prime}} .
$$


Poming aloris

$$
y:=\frac{1}{a(l)} y^{\prime}, z:=\frac{1}{y^{\prime}(l)}:
$$

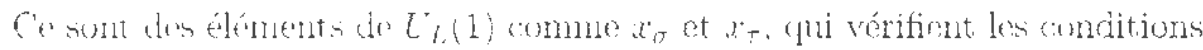

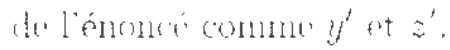

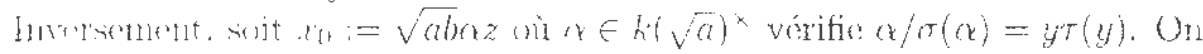
: 1

$$
\frac{(r) x(0)}{x_{0}}=\frac{1}{y^{2}} \cdot \frac{\tau(x)}{x_{0}}=\frac{1}{z^{2}} \cdot \frac{\pi \tau\left(x_{0}\right)}{x_{0}}-\frac{1}{(y \sigma(z))^{2}} \text {. }
$$

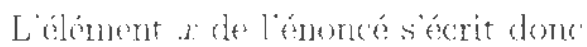

$$
x=\theta+\frac{x_{0}}{\sigma\left(x_{0}\right)} \sigma(\theta)+\frac{r_{0}}{\tau\left(x_{1}\right)} \tau(\theta)+\frac{r_{0}}{\sigma \tau\left(x_{1}\right)} \sigma \tau(\theta) .
$$

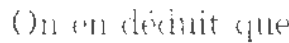

$$
(x) / x=\gamma\left(x_{0}\right) / x_{0} \quad(n \in \operatorname{Col}(L / k))
$$

D'aprisi lo (3.5) de 10 , ceci implique en particulier que l'extension $\lambda=L(\sqrt{x}) / h$ s qut quarnionjenne comme $L\left(\sqrt{x}_{0}\right) / k$. Enfin, les éléments $y$ *t z cituint dalls $U_{h}(1)$, leurs carrés sont dans $U_{L}(2)$, et conme $\operatorname{Tr}_{L / k}(\theta)=1$ . wis a biens $x \in L_{i,(2)}$.

La délnonstration du théorème 1.1 dans le cas modéré utilisera les denx lemmes qui suivent.

Pulur tout cycle $\mathcal{M}=\mathcal{M}_{\infty} \mathcal{M}_{0}$ de $k$ où $\mathcal{M}_{\infty}=\prod_{\sigma(k) \subset \mathbf{R}} \sigma, \mathcal{M}_{0}$ étant un idtral outier do $O_{k}$. on désigne par $C l(b, \mathcal{M})$ le groupe des classes de rayun moluln, M. Rappelons que dans les notations de la theorie du corps de clitsbers (cf.j1). Chap.II), on écril $a \equiv 1 \bmod ^{*}(\mathcal{M})$, avec $a \in k^{x}$, si et sulument si :

(1) quel que soit $\sigma \mid \mathcal{M}_{\infty}, \sigma(a)>0$

(2) quel que soit $B \mid \mathcal{M}_{0}, v_{\mathcal{B}}(a-1) \geq v_{\mathcal{B}}\left(\mathcal{M}_{0}\right)$, où v désigne la valuation I'] $B$.

Lerrme 25 (1) Pour tout $c \in \mathcal{C} l(k)$ il existe:

- $m \in k^{\times}$tel que $m \equiv 1 \bmod ^{*}\left(\mathcal{M}_{\infty} 4 O_{k}\right)$

- $B$ un idéal premier de $O_{k}$ ne divisant pas $2 O_{k}$

- I un idcal fractioniaire de $O_{k}$ 
nitis gite

$$
m O_{k}=I^{2} B . \quad r=\mathrm{Cl}(I)^{-1} .
$$

(2) I'our lî̀céal premier $B$ du (1). il existe $m^{\prime} \in k^{x}$, et un jdéal prenicr $\mathcal{B}^{\prime} \neq \mathcal{B}$ do $O_{k}$ ne divisant pas $2 O_{k}$. tels que

$$
m^{\prime} O_{k}=\mathcal{B}^{\prime} . \quad m^{\prime} \equiv 1 \bmod ^{*}\left(\mathcal{M}_{\infty} 4 \mathcal{B}\right) .
$$

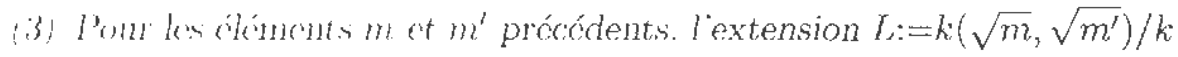
ast biscrique biquadratique et modérément ramifiée.

Dómonstration. (1) Considérons $r^{-1} \in \mathcal{C}(k)$ et le cycle $\mathcal{M}:=\mathcal{M}_{\infty} 4 O_{k}$. Dapros la surjection canonique de $\mathcal{C} l(k, \mathcal{M})$ sur $C l(k)$ (cf.[7, p.125), et lo thóneme do densité génćralisé de Dirichlet (cf.[1], p.27), il existe un idferal fractiomaire dr $O_{k}$, ot un idéal premier $\mathcal{B}$ de $O_{k}$ ne divisant pas 20 . Lels qur $r^{-1}=\mathcal{C l}(I)$ ot $\mathcal{C l}(I)^{-2}=\mathcal{C l}(\mathcal{B})$. L'existence d'un élément. $m \equiv 1 m o d^{*}(\mathcal{M})$ vérifiant l'égalité du (1) est donc claire.

Pour lat chímonstration du (2). on consiclère lat classe triviale de $\mathcal{C} l(k)$ el

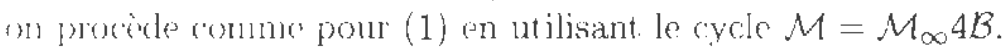

(3) $\mathrm{DO} m \mathrm{O}_{k}=I^{2} \mathrm{~B}$ a $\mathrm{m}^{\prime} \mathrm{O}_{k}=\mathcal{B}^{\prime}$. on déduit que $\mathrm{u}_{\mathfrak{B}}(\mathrm{m}) \equiv 1 \bmod 2$ " $l k^{\prime}\left(m^{\prime}\right)=1$. Donc $m$ ct $m^{\prime}$ ne sont pas des carrés clans $k$, de sorte qur los axtenion $h(\sqrt{m}) / k$ et $k\left(\sqrt{m^{\prime}}\right) / h$ sont quadratiques. Comme $m$ et $m^{\prime}$ wont congrus à 1 mod* $\left(4 O_{k}\right)$, ces extensions sont modérément ramifiées de discriminants respectifs $\mathcal{B}$ et $\mathcal{B}^{\prime}$ en vertu de la proposition 2.1. On a $B \neq \mathcal{B}^{\prime}$ : simon. lat congruence que vérifie $m^{\prime}$ entrainerait $m^{\prime}-1 \in \mathcal{B}$, d'où $1 \in \mathcal{B}$. cr qui est inpussible. Par suite $k(\sqrt{ } m i) \neq k\left(\sqrt{m^{\prime}}\right)$. La composéc de $h(\sqrt{m}) / h$ et $h \cdot(\sqrt{m}) / h$ est donk bicyclique biquadratique ot modórément rituilicos.

On a le corollaire suivant du thésrème 1.2 et du (1) du lemme 2.5 .

Corollaire 2.6. Pour tout corps de nonbres $k$, l'ensemble des éléments de $C l(k)$ rólisables connnc classes de Steinitz d'extensions quadratiques de $k$, mulcimont vanifiées of plongeables dans dos cxtensions quaternioniemes

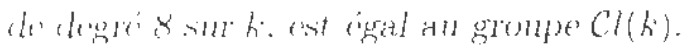

Dimomstraton. Soit $\quad \in C l(h)$. Dans les notations du lermme 2.5 et en vertu de la proposition 2.1, c est la classe de Steinitz de l'anneau des entiers de lextention $h(\sqrt{m}) / k$. Conne $m$ est totalement positif, toute place réelle do $h$ so prolonge en une place réelle de $k(\sqrt{m})$. On conclut alors grâce au thim 
Lemme 27 Lixtention $L \cdots H\left(\sqrt{m}, \sqrt{m^{2}}\right) / h$ du lenme 2.5 so plonge dants une oxterlsion $\mathrm{V} / \mathrm{h}$ quaternionientue de degré 8 nodérénent ramifiée.

Démonstration. On sait que $L / k$ est plongeable dans une extension quaternionifme de degre 8 si et seulcment si pour toute place $y$ de $k$. on a la conditim $\left(^{*}\right)(-1 . m)^{\prime}\left(-1 . m^{\prime}\right)_{t^{\prime}}\left(m, m^{\prime}\right)_{t^{\prime}}=1$ oin pour tous $a, b \in k^{\times},(a, b)_{v}$

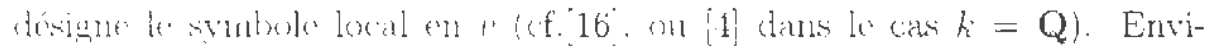

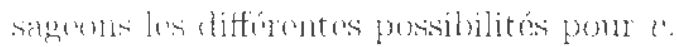

(1) Si $u^{\prime}$ cot complexo. (*) est rérifice par défintion de $(a, b)_{2}$. Si $v$ est réclle. $\left({ }^{*}\right)$ csst vérifiéc car $m$ et $m^{\prime}$ sont totalennent positifs en vertu desc compruences du lennue 2.5 .

12) Sivent $T$ un ichal preniver do $O_{k}$ au clessus de 2 et $e$ son indice de ranuificat ion absulu. Les congruences que vérifient $m$ et $m^{\prime}$ entrainent $l^{\prime} n(m-1) \geq l^{\prime}\left(1 O_{k}\right)=20$ et de même $v_{\mathcal{D}}\left(m^{\prime}-1\right) \geq 2 e$. D'après ([1] : p.237.Prop.6), il existe deux entiers $a_{1}, a_{2} \in \mathbb{Z}$ tels que

$$
\begin{gathered}
\left(-1, m_{\mathcal{D}}=(-1)^{v_{\mathcal{D}}(-1) a_{1}}, \quad\left(-1, m^{\prime}\right)_{\mathcal{D}}=(-1)^{\nu_{\mathcal{D}}(-1) a_{2}}\right. \\
\left(m, m^{l}\right)=(-1)^{v_{\mathcal{D}}(m) a_{2}}
\end{gathered}
$$

e1 cés symboles sont tous égaux à 1 car $v_{D}(m) \equiv 0 \bmod 2$.

(3) Soit $\mathcal{P}$ un idéal premier de $O_{k}$ qui n'est pas au dessus de 2. Soient $k_{p}$ le complité de $k$ on la place $\mathcal{P}$ et $\overline{k_{p}}$ son corps résiduel de cardinal $q$. Prurr tuls a $b \in h^{\times}$pusons $c:=(-1)^{\alpha \beta} a^{\beta} b^{-\alpha}$, où $\alpha:=u_{p}(a), \beta:=$

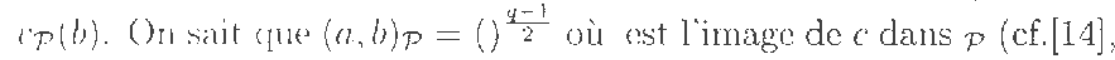

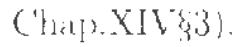

(3.1) On suppose que $P \neq B$ at $\mathcal{P} \neq \mathcal{B}^{\prime}$. D'après le lemme 2.5 , on a i $(m) \equiv 0 \bmod 2$ et $v_{\mathcal{p}}\left(m^{\prime}\right)=0$. Si $a=-1$ et $b=m$, on a $\checkmark=(-1)^{m_{\mathcal{P}}(m)}=1$, diòi a fortiori, $\left(-1, m_{\mathcal{P}}=1\right.$. De mêmo $\left(\cdot 1 . m^{\prime}\right)_{p}=1$. Si $a=m$ et $b=m^{\prime},=\bar{m}^{-v_{p}(m)}$ et $\left(m n, m n^{\prime}\right)_{p}=$ $\left.\left(\overline{m^{\prime}}\right)^{\prime \prime}\right)^{\frac{(p(m)}{2}}=1$.

(3.2) Pill la fornule du produit, il suffit maintenant d'examiner le cas $\mathcal{P}=\mathcal{B}$. Pour $a=m$ et $b=m^{\prime}$, comme $v_{\mathcal{B}}\left(m^{\prime}\right)=0$, il vient $c=m^{\prime v_{B}(m)}$. Or la congruence $m^{\prime} \equiv 1 \bmod ^{*}\left(\mathcal{M}_{\infty} 4 \mathcal{B}\right)$ se traduit dans $B$ par $\overline{m^{\prime}}=$, doù $\left(m, m^{\prime}\right)_{B}=1$. Ponr $a=-1$ ct $b=m^{\prime}$ on à $c^{\prime}=(-1)^{m_{B}\left(m^{\prime}\right)}=1$, d'où $\left(-1, m^{\prime}\right)_{B}=1$. Si $a=-1$ (f $b=m \cdot r=(-1)^{r(B)}=-1$ car $\iota_{B}(m) \equiv 1$ mod 2 . d'où 
$(-1 . m)_{\mathcal{B}}=(-1)^{\frac{q-1}{2}}$. Or. diune part $N_{k / \mathbf{Q}}(\mathcal{B})=q$, d'autre part $\mathcal{B}=\Delta(k(\sqrt{m}) / k)$. Comme $\mathcal{B}$ est premier avec $2 O_{k}$, sa norme est inpaire: de plus, aucune place réelle de $k$ ne se prolonge en une place complexe de $k(\sqrt{m})$. Le théorème 1.4 de [9] nous dit alors que $N_{k / \mathbf{Q}}(\mathcal{B})=(-1)^{0} \bmod 4$, i.e $q \equiv 1 \bmod 4$. Par suite $(-1 . m)_{\mathcal{E}}=1$. Ceci achève la démonstration du lemme 2.7 .

\section{Démonstration du théorème 1.1}

(1) Le cas modéré

Soit c un élément quelconque de $\mathcal{C l}(k)$. Considérons l'extension du lemme 2.7: $L=k\left(\sqrt{m}, \sqrt{m^{\prime}}\right) / k$. D'après ([12]. Theorem 6.6) ou [11], elle est plongeable dans une extension quaternionienne de degré 8 modérément raunifié. ()r pour l'oxtension $K=k(\sqrt{m}) / k$, on a $c=\mathcal{C} l\left(O_{K}\right)$ on vertu de la

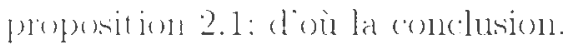

(2) Le cas salurage

Soicnt $r \in \mathcal{C} l(k)$ et $J_{1}$ le produit des idéaux premiers de $O_{k}$ au dessus de 2. $\mathrm{D}$ après le théorène de densité généralisé de Dirichlet, il existe d'une part un idéal premier $\mathcal{B}_{1}$ de $O_{k}$ ne divisant pas $2 O_{k}$, d'autre part un idéal premier $\mathcal{B}_{2}$ de $O_{k}$ premier arec $2 \mathcal{B}_{1}$, tels que $c^{-1}=\mathcal{C l}\left(\mathcal{B}_{1}\right)$ et $c^{2} \mathcal{C l}\left(J_{1}^{-1}\right)=\operatorname{Cl}\left(\mathcal{B}_{2}\right)$ dans $C l^{+}\left(k_{i}\right)$ le groupe des classes de $h^{\prime}$ au sens restreint. D'où l'existence d'un

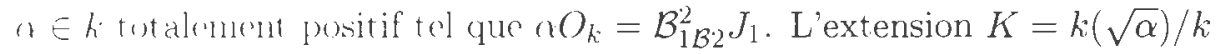
ust clladratique (ar ${ }^{\prime} \mathcal{B}_{2}(\alpha)=1$. D'après la proposition 2.1 , les idéaux premiers $\mathcal{P}$ de $O_{k}$ ramifiés dans $K$ sont $\mathcal{B}_{2}$ et tout idéal premier de $O_{k}$ au dessus de 2 . Pour de tels $\mathcal{P}$. on a v' $\mathcal{P}(\alpha)=1$; par suite le polynôme $X^{2}-\alpha$ est un polynome d'Eisenstein dans $k_{\mathcal{P}}[X]$, et $\{1, \sqrt{\alpha}\}$ est une base locale d'entiers. Donc $v_{p}\left(\Delta\left(K / k^{\prime}\right)\right)=v_{p}(4 \alpha)=1+v_{\mathcal{P}}\left(4 O_{k}\right)$. On en déduit que $\Delta\left(K^{\circ} / h^{\prime}\right)=\mathcal{B}_{2} J_{1}\left(2 O_{k}\right)^{2}$. d'où $\mathcal{C l}\left(O_{k^{*}}\right)=\mathcal{C l}\left(\mathcal{B}_{1}^{-1} 2^{-1} O_{k}\right)=c$ en vertu de la proposition 2.1. Pour tout idéal premier $\mathcal{D}$ de $O_{k}$ au dessus de $2,-\alpha$ ne peut itre un carré dans $k_{D}$ car $v_{D}(\alpha)=1$. Comme $\alpha$ est totalement positif, on cn déduit par '2] que a est somme de trois carrés, donc que l'extension saluvagement ramifiée $K / k$ se plonge dans une extension quaternionienne de degre 8 . Remarquons enfin que pour la classe triviale $c=1$ de $\mathcal{C l}(k)$, il ni est par nécessisare de prendre pour $\mathcal{B}_{1}$ un idéal premier; il suffit de prendre $\mathcal{B}_{1}=()_{k}$.

\section{3.. Exemples}

Soit $k=\mathrm{Q}(\sqrt{85})$. Pour tout entier premier $p, \mathcal{B}_{p}$ désigne un idéal premier de $O_{k}$ : 11 dessits de $p$. Le nombre de classes de $k$ est 2 et son unité fonda- 


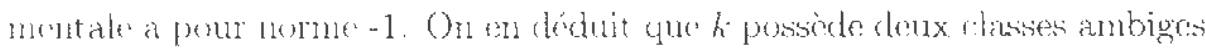
"to que $C l(k)=\left\{1, C l\left(B_{1}\right)\right\}=\left\{1 . C /\left(B_{5}\right)\right\}$

\section{Le cas nodéré}

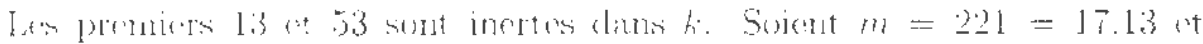
$m i$.

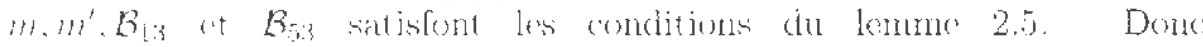

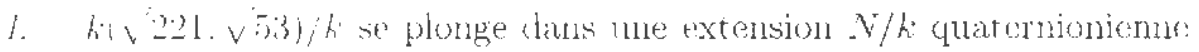
muláment ranifice de degré 8 . Solent les extensions quadratiques $h_{1}:=h(\sqrt{221}) / h$ ot $h:=h(\sqrt{53}) / h$ Le môno lomme et la proposition 2.1 domlat $C l\left(O_{K_{1}}\right)=\mathrm{Cl}\left(\mathrm{B}_{1}-\right)$ at $\mathrm{Cl}\left(\mathrm{O}_{K_{2}}\right)=1$.

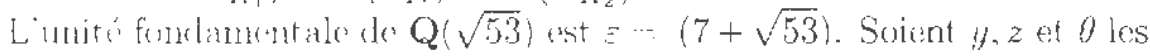

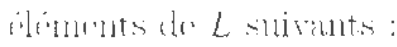

$$
\begin{gathered}
y=(\sqrt{221}-2 \sqrt{53}) \\
z=\varepsilon^{3}(\sqrt{221}-2 \sqrt{53})=(182+25 \sqrt{53})(\sqrt{221}-2 \sqrt{53}) \\
\theta=(1+\sqrt{221}+\sqrt{53}+\sqrt{221.53}) .
\end{gathered}
$$

En uilimant lom rosiltats de $[17 j$ dombant la forme dos ontiers de $\mathrm{Q}(\sqrt{22} \cdot \sqrt{53})$, w1 montre que $y, z \in U_{L}(1)$ et que $\theta$ est $u n$ entier de $L$. On veiptie facilement que y et $z$ satisfont les conditions (2.1) du théorème. 1.2. Commu la 1 rás de $\theta$ sur $k$ est égale à 1 , on obtient par le (2.2) de ce muruc thencruse que lextension $N=L(\sqrt{x}) / h$ oi

$$
r=\theta+y^{2} \pi(\theta)+z^{2} \tau(\theta)+(y \sigma(z))^{2} \sigma \tau(\theta)
$$

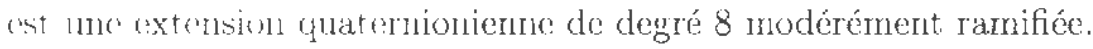

\section{Le cas sauvage}

On utilise la mothoute de la détuonstration du théorème 1.1 dans le cas

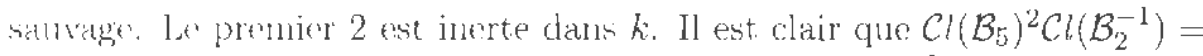

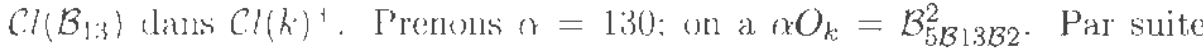
$h_{1}:=h(\sqrt{a}) / h$ est quadratique, sauvagenont ramifiée, plongeable dans unu uxtension guaternionienne $N_{1} / \mathrm{h}$ de degré 8 el l'on a $\mathrm{Cl}\left(\mathrm{O}_{K_{1}}\right)=\mathrm{Cl}\left(\mathcal{B}_{5}\right)$. Do merme on rerifie facilement que l'exterision $K_{2}:=k(\sqrt{26}) / k$ est sauvage, plomgrable dam un oxtemsion quaternionienme $N_{2} / h$ de degré 8 , et que $\left.\mathrm{Cl}()_{1,2}\right)=1$. 


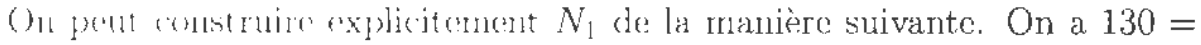
$3^{2}-6 j^{2}-(\sqrt{85})^{2}$. Comns 45 ot 130.45 ne sont pas des carrés dans $k$, on ubtient par $[5]$ que l'extension $L=k(\sqrt{5}, \sqrt{130})$ est plongeable dans l'exterision quaternionienne $N_{1}=L(\sqrt{x})$ de degré 8 sur $k$, où

$$
x=5 \sqrt{26}(3 \sqrt{5}+\sqrt{130})(\sqrt{5}+1) .
$$

Renurquons quet la lroisiènue sous-extension quadratique de $L$ est $k(\sqrt{26})$. On pent donc prendre $N_{2}=N_{1}$.

\section{Remerciements}

Lis mutcurs remerciont Michel Korvaire pour son hospitalité à l'Université de Gencive. 


\section{Références}

1' Ph. (ASSOL-NOGLES and M.J. TAYLOR, Elliptic Functions and Rings of Integers. Birkhaüser, Boston, (1987).

[2] P. DAMEY el J. MARTINET, Plongement dine extension quadra-

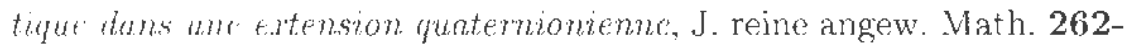
263. pr. 323-338. (1973).

3. A. FIOHLLC The discrimitants of relative extensions and the exi.tene of integrol bases, Mathematika 7, pp. 15-22, (1960).

[4] A. FRÖIILICII, Artin-rool numbers and normal integral bases for quale rmon fields, Invent. Math. 17, pp. 143-166, (1972).

[5] G. FUJISAKT. An elementary constrution of Galois quaternion ex4h nism. Proc. Japrn Acad. 66A, pp. 80-83, (1990).

[6] L. JWChE, Lectures on the Theory of Algebraic Numbers, Graduate Texts Math. 77. Springer-Verlag: New York: (1981).

7) S. LANG. Algebraic Nunber Theory. Graduate Texts Math. 110, Springer-Verlag. New York. 1986.

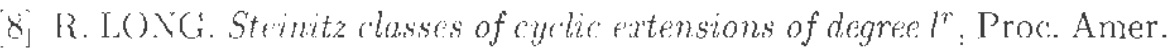
Nitth. Sxk. 49. pr. 297-304, (1975).

9] J. MARTINET. Les discriminants quadratiques el la congruence de Stretrlberyer. Sein. Theor. Nombres Bordeaux 1, pp. 197-204, (1989).

[10] R. 11 $155 \mathrm{Y}$, Construction de p-extensions goloisiennes d'un corps de mantintique differente de p. J. Nlgebra 109, pp. 508-535, (1987).

IIj R. MASSY. Bases nomales d'entiers relatives quadraliques, J. Number Theory 38, pp. 216-239, (1991).

12] J. NEUKIRCH. Über das Eirbettungsproblem der algobraischen Zuhlentherte, Invent. Math. 21, pp. 59-116, (1973).

1:3. F. ROSLNBLCTH. Die arthantische Theorte und Konstruktion (4) Quatomincnkiörper auf Klassenkörpertheorelischer Grundlage, Mondsth. Alath. Phys. 41, pp. 85-125, (1934). 
[14] J.-JP. SFRRF. Corps Iocaux, 3è éd., Hermann, Paris, (1980).

[15] B. SOGAÏGUI, Structure galoisienne relative des anneaux d'entiers, J. Nunber Thery 28, pp. 189-204, (1988).

[16] E. WITT, Konstruktion von galoisschen Körpern der Charakteristik $p$ zu vorgegebener Gruppe der Ordnung $p^{f}$, J. Crelle 174, pp. 237-245, (1936).

[17] K.S. WILLIAMS, Integers of biquadratic fields, Canad. Math. Bull. 13(4). pp. 519-526. (1970).

Received: June 10, 1996.

Richard Malssy

(4)

Bomethaib) Sulaigni

Départenent de Mathématiques

Université de Valencienres

Le Mont Houy, BP311, F-59304

Valenciennes

France 